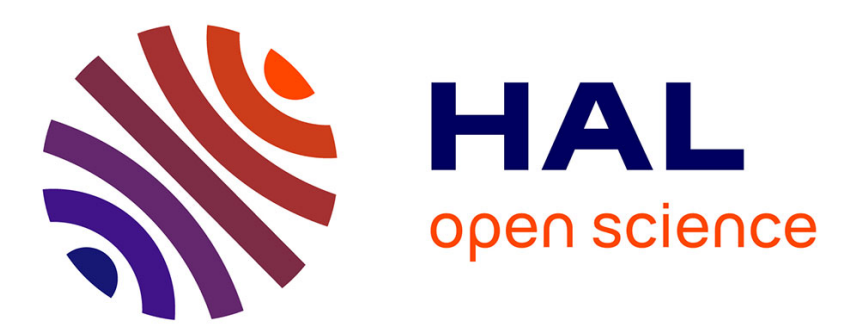

\title{
Geometrical Patterns for Measurement Pose Selection in Calibration of Serial Manipulators
}

\author{
Alexandr Klimchik, David Daney, Stéphane Caro, Anatol Pashkevich
}

\section{To cite this version:}

Alexandr Klimchik, David Daney, Stéphane Caro, Anatol Pashkevich. Geometrical Patterns for Measurement Pose Selection in Calibration of Serial Manipulators. Lenarčič, Jadran and Khatib, Oussama. Advances in Robot Kinematics, Springer International Publishing, 2014, 978-3-319-06697-4. 10.1007/978-3-319-06698-1_28. hal-01004166

\section{HAL Id: hal-01004166 \\ https://hal.inria.fr/hal-01004166}

Submitted on 17 Mar 2019

HAL is a multi-disciplinary open access archive for the deposit and dissemination of scientific research documents, whether they are published or not. The documents may come from teaching and research institutions in France or abroad, or from public or private research centers.
L'archive ouverte pluridisciplinaire HAL, est destinée au dépôt et à la diffusion de documents scientifiques de niveau recherche, publiés ou non, émanant des établissements d'enseignement et de recherche français ou étrangers, des laboratoires publics ou privés. 


\title{
Geometrical patterns for measurement pose selection in calibration of serial manipulators
}

\author{
Alexandr Klimchik ${ }^{a}$, David Daney ${ }^{b}$, Stephane Caro ${ }^{c}$ and Anatol Pashkevich ${ }^{a}$
}

\begin{abstract}
The paper is devoted to the accuracy improvement in geometric calibration of serial manipulators. Particular attention is paid to the optimal selection of measurement poses, which reduce measurement noise impact on the parameters identification precision. In contrast to previous works, the proposed approach yields simple geometrical patterns that allow user to take into account the joint and workspace constraints and to find measurement configurations without tedious computations. The advantages and practical significance of the proposed approach are illustrated by an example that deals with 6 -dof serial manipulator.
\end{abstract}

Key words: calibration, design of experiments, geometrical patterns, serial robot.

\section{Introduction}

Since most of industrial serial robots operate without position feedback, the precision of a geometric model used in the control algorithm should be high enough. In order to meet this requirement in practice, the calibration technique is usually applied. For this reason, the problem of robot calibration has always been in the focus of the robotic community. There exist open-loop and closed-loop techniques that are suitable for serial and parallel manipulators $[3,4]$. However, to apply them in a real industrial environment, it is necessary to reduce the impact of the measurement noise that corrupts the input data and is evaluated via the terms of the covariance matrix [6].

\footnotetext{
${ }^{a}$ Ecole des Mines de Nantes, IRCCyN, France

${ }^{b}$ Institut National de Recherche en Informatique et en Automatique, INRIA, France

${ }^{c}$ Centre National de la Recherche Scientifique, France

e-mail: alexandr.klimchik@emn.fr, david.daney@inria.fr, stephane.caro@irccyn.ec-nantes.fr, anatol.pashkevich@emn.fr
} 
In order to reduce the impact of measurement noise, two approaches can be generally applied: (i) increasing the number of experiments and (ii) applying design of experiment theory for selection of measurement configurations. The first approach is quite a simple, it does not require any computation, but increases a lot the measurement time and related cost. In contrast, the second approach allows us to increase identification accuracy without increasing the number of measurements [2]. However this approach requires additional knowledge and some computational efforts. Nevertheless, because of its advantages the second approach is more attractive and is in the focus of this paper.

Although the design of experiment theory has been used for more than a century in different areas, the obtained results cannot be applied directly in robotics since they are mostly suitable for linear regression models. Therefore, to obtain similar results for robot calibration that rely on highly non-linear models, the existing techniques should be essentially revisited. In addition, to make the technique attractive for practicing engineers, it is reasonable to obtain some geometrical patterns allowing the user to generate optimal measurement configurations without any computational efforts.

\section{Problem of measurement pose selection in robot calibration}

To show the importance of the problem of interest, let us present first a simple motivation example. For the purpose of simplicity, let us limit our study by a 2-dof planar manipulator with two actuated revolute joints and link lengths $l_{1}=1.0 \mathrm{~m}$, $l_{2}=1.0 \mathrm{~m}$. This manipulator has four geometrical parameters to be identified: (i) two link length deviations and (ii) two joint encoder offsets.

For comparison purposes, let us examine two sets of measurement configurations assuming that the measurement noise is Gaussian with zero mean and the standard deviation $\sigma=0.1 \mathrm{~mm}$. Simulation results are summarized in Fig. 1, where the rootmean-square errors $\rho$ of the end-effector position after calibration throughout the robot workspace are presented. As follows from Fig. 1, in the worst manipulator configuration, the Set \#1 provides a positioning error equal to $2.29 \mathrm{~mm}$ while the Set \#2 reduces the worst positioning error down to $0.14 \mathrm{~mm}$, i.e. by a factor of 16. Hence, this simple example clearly shows that the selection of measurement configurations is a very important issue in robot calibration. In fact, poorly chosen measurement configurations may have a negative effect and even reduce the robot accuracy after calibration. This motivates careful planning of the calibration experiments in order to increase calibration efficiency, which is in the focus of the paper.

In the frame of this paper, a general planar manipulator with revolute joints is considered, whose the end-effector position is computed using the following expressions

$$
x=\sum_{i=1}^{n}\left(l_{i}^{0}+\Delta l_{i}\right) \cdot \cos \left(\theta_{i}^{0}+\Delta \theta_{i}\right) ; \quad y=\sum_{i=1}^{n}\left(l_{i}^{0}+\Delta l_{i}\right) \cdot \sin \left(\theta_{i}^{0}+\Delta \theta_{i}\right)
$$




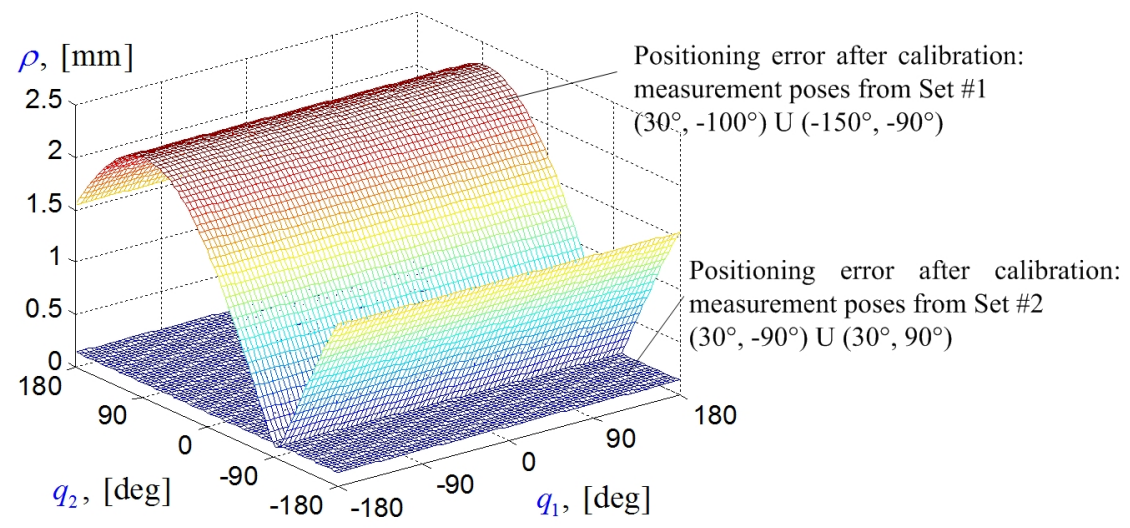

Fig. 1 Manipulator accuracy after calibration for two different sets of measurement poses.

where $l_{i}^{0}$ are the nominal link lengths, $\Delta l_{i}$ are their deviations, $q_{j}^{0}$ are nominal joint coordinates, the variables $\theta_{i}^{0}$ are defined as $\sum_{j=1}^{i} q_{j}^{0}, \Delta \theta_{i}$ are the joint offsets and $n$ is the number of links. Collecting the unknown parameters $\left(\Delta l_{i}\right.$ and $\left.\Delta \theta_{i}\right)$ into the vector $\Delta \pi$ and the measurements $\left(x^{k}, y^{k}\right)$ into the vector $\Delta \mathbf{P}^{k}$, expression (1) can be rewritten as $\Delta \mathbf{P}=\mathbf{J} \Delta \pi$, where $\mathbf{J}$ is a Jacobian matrix. Then, one can get unknown parameters using the least-square technique that leads to

$$
\Delta \pi=\left(\sum_{k=1}^{m} \mathbf{J}^{k^{T}} \mathbf{J}^{k}\right)^{-1} \cdot \sum_{k=1}^{m} \mathbf{J}^{k^{T}} \Delta \mathbf{P}^{k}
$$

where the superscript ${ }^{\prime} k$ indicates the experiment number, and $m$ is the number of measurements.

Taking into account that each measurement is corrupted by the unbiased random Gaussian noise with standard deviation (std) $\sigma$, the identification accuracy of the parameters $\Delta \pi$ can be evaluated via the covariance matrix, which is computed as follows:

$$
\operatorname{cov}(\Delta \pi)=\sigma^{2}\left(\sum_{k=1}^{m} \mathbf{J}^{k^{T}} \mathbf{J}^{k}\right)^{-1}
$$

The latter allows us to evaluate the impact of measurement noise on the parameters identification accuracy and can be used to estimate the quality of measurement configurations. Based on the covariance matrix, it is possible to choose the measurement configurations that yield parameters less sensitive to measurement noise. In engineering practice, this procedure is referred to as the design of calibration experiments. In order to compare different plans of experiments (i.e. their efficiency), different performance measures have been proposed that deal with the norm, trace, etc. of the covariance matrix or information matrix (its inverse) [1]. In [5] the authors proposed an optimality condition of the calibration plan where the main idea 
is to ensure that the information matrix is diagonal. This condition corresponds to the D-optimal plan of experiments and is satisfied when

$$
\sum_{k=1}^{m} \cos \left(\sum_{s=j}^{i} q_{s}^{k}\right)=0, \quad \sum_{k=1}^{m} \sin \left(\sum_{s=j}^{i} q_{s}^{k}\right)=0, \quad \forall i, j=\overline{2, n}, \quad i \geq j
$$

i.e. the sums of sines and cosines of links orientation $q_{s}^{k}$ with respect to any joint $s$ should be equal to zero.

It has been proved that the above presented equations define the desired set of the optimal measurement configurations, but in general case solution of this system requires essential efforts. In fact, for $n=2, m \geq 2$ expressions (4) provide two simple constraints only $\sum_{k=1}^{m} \cos q_{2}^{k}=0, \sum_{k=1}^{m} \sin q_{2}^{k}=0$, which are imposed on the $2 m$ design variables $\left\{q_{1}^{k}, q_{2}^{k} \mid k=\overline{1, m}\right\}$. So, relevant configurations can be easily found geometrically [5]. However, even in the case of $n=3, m \geq 3$ there are 6 rather complicated trigonometric constraints imposed on $3 m$ design variables $\left\{q_{1}^{k}, q_{2}^{k}, q_{3}^{k} \mid k=\overline{1, m}\right\}$. Therefore, the solution of the corresponding underconstrained system of algebraic equations becomes non-trivial. It is apparent that the complexity of the problem essentially increases with $n$ (the number of manipulator links). Additional difficulties arise when the joint limits should be taken into account (i.e. $\left\{q_{i}^{\min } \leq q_{i}^{k} \leq q_{i}^{\max } \mid i=\overline{1, n} ; k=\overline{1, m}\right\}$ ). Hence, it looks reasonable to develop relevantly simple and efficient technique allowing practical engineers to select desired measurement configurations in accordance with equations (4) without any tedious computations. This problem is in the focus of this paper.

\section{Geometrical patterns for measurement pose selection}

Before defining patterns, let us obtain some important properties of the optimality conditions (4) that allow us to reduce the problem complexity.

Property 1: Superposition of optimal plans gives also an optimal plan for this.

Proof of this property is obvious and is based on the additivity of the operations included in (4). Using this property it is possible to generate optimal plans with a large number of measurement configurations using simple sub-sets. In a trivial case, it is possible to repeat experiments using the same set of optimal configurations. The latter is very important in practice, since it is much less time consuming to repeat experiments without changing manipulator configurations.

Property 2: The angles $q_{2}, \ldots q_{n}$ can be rearranged in the optimal plans in an arbitrary manner without losing the optimality conditions (4).

Proof. Let us assume that for an n-link manipulator optimal plan consists of $m$ measurement configurations. Let us now change in the optimal plan column $a$ and $b$ $(a, b>1)$ and analyze the sets of angles for which sums of sines and cosines should be satisfied in the optimal plan $\sum_{s=j}^{i} q_{s}^{k}$. In this case conditions (4) will be satisfied for $i, j=\overline{2, a}$ and $i, j=\overline{b, n}$ since all arguments of sines and cosines remain the same. For case $a \leq j \leq i \leq b$ conditions also do not change. However, in the cases $a \leq j \leq$ 
$b \leq i$ and $j \leq a \leq i \leq b$ optimality conditions $\sum_{s=j}^{i} q_{s}^{k}$ will be replaced $\sum_{s=j}^{i} q_{s}^{\prime k}=$ $\sum_{s=j}^{i} q_{s}^{k}+q_{a}^{k}-q_{b}^{k}$ by and $\sum_{s=j}^{i} q_{s}^{\prime k}=\sum_{s=j}^{i} q_{s}^{k}+q_{b}^{k}-q_{a}^{k}$ respectively, where prime indicates the angle corresponding to the rearranged new set of angles. Assuming that plan $\sum_{s=j}^{i} q_{s}^{k}$ is optimal, plans $\sum_{s=j}^{i} q_{s}^{\prime k}-q_{a}^{k}+q_{b}^{k}$ and $\sum_{s=j}^{i} q_{s}^{\prime k}+q_{a}^{k}-q_{b}^{k}$ will be also optimal. Further, taking into account that the superposition of optimal plans gives optimal plan and applying it to the last two plans gives plan $2 \sum_{s=j}^{i} q_{s}^{\prime k}$ that should be also optimal. This plan can be split into two sub-plans $\sum_{s=j}^{i} q_{s}^{\prime k}$ for which the sums of sines and cosines should be equal. Taking into account that double sum is equal to zero, the plan $\sum_{s=j}^{i} q_{s}^{\prime k}$ will be also optimal.

This property can be useful when the joint limits are narrower than the minimum range required for the optimal plan. In this case, it is reasonable to change joint coordinates with the ones where joint limits are not so critical (more than $240^{\circ}$ ) and where the required range of joint variations is lower.

Property 3: Optimal plan for n-link manipulator can be obtained using two lower-order optimal plans for $n_{1}$ - and $n_{2}$-link manipulators, where $n_{1}+n_{2}=n+1$.

This property gives us an elegant technique to generate optimal plans of calibration experiments without straightforward solution of system (4). In this case the number of measurement configurations is defined by $n_{1} \times n_{2}$. In fact, by a sequential splitting of the original $n$-link kinematic chain, it is possible to reduce the problem complexity and to replace the problem of generating optimal plan for $n$-link manipulator by several sub-problems for 2-, 3- and 4-link manipulators. The latter motivates us to develop typical geometrical patterns that can be used directly to generate optimal plans for complex manipulators.

Now let us introduce some geometrical patterns for typical serial manipulators that can be used to generate optimal plans. In the frame of these patterns, all variables $\alpha_{i}, \beta_{i}$ and $\gamma, \delta$ are treated as arbitrary angles.

For $n=2, m=2$, the complete set of solutions can be expressed as:

$$
\begin{aligned}
& q_{1}^{1}=\alpha_{1} ; \quad q_{2}^{1}=\beta \\
& q_{1}^{2}=\alpha_{2} ; \quad q_{2}^{2}=\beta+\pi
\end{aligned}
$$

For $n=2, m=3$, the desired set of solutions is

$$
\begin{array}{lc}
q_{1}^{1}=\alpha_{1} ; \quad & q_{2}^{1}=\beta \\
q_{1}^{2}=\alpha_{2} ; & q_{2}^{2}=\beta+2 \pi / 3 \\
q_{1}^{3}=\alpha_{3} ; & q_{2}^{3}=\beta-2 \pi / 3
\end{array}
$$

For $n=3, m=3$, the geometrical pattern can be presented as

$$
\begin{array}{lcc}
q_{1}^{1}=\alpha_{1} ; & q_{2}^{1}=\beta ; & q_{3}^{1}=\gamma \\
q_{1}^{2}=\alpha_{2} ; & q_{2}^{2}=\beta+2 \pi / 3 ; & q_{3}^{2}=\gamma+2 \pi / 3 \\
q_{1}^{3}=\alpha_{3} ; & q_{2}^{3}=\beta-2 \pi / 3 ; & q_{3}^{3}=\gamma-2 \pi / 3
\end{array}
$$

For $n=3, m=4$, an optimal solution can be expressed as 


$$
\begin{array}{lcc}
q_{1}^{1}=\alpha_{1} ; & q_{2}^{1}=\beta_{1} ; & q_{3}^{1}=\gamma \\
q_{1}^{2}=\alpha_{2} ; & q_{2}^{2}=\beta_{1}+\pi ; & q_{3}^{2}=\gamma \\
q_{1}^{3}=\alpha_{3} ; & q_{2}^{3}=\beta_{2} ; & q_{3}^{3}=\gamma+\pi \\
q_{1}^{4}=\alpha_{4} ; & q_{2}^{4}=\beta_{2}+\pi ; & q_{3}^{4}=\gamma+\pi
\end{array}
$$

For $n=4, m=4$, the desired geometrical pattern can be written as

$$
\begin{array}{lccc}
q_{1}^{1}=\alpha_{1} ; & q_{2}^{1}=\beta_{1} ; & q_{3}^{1}=\gamma ; & q_{4}^{1}=\delta \\
q_{1}^{2}=\alpha_{2} ; & q_{2}^{2}=\beta_{1}+\pi ; & q_{3}^{2}=\gamma ; & q_{4}^{3}=\delta+\pi \\
q_{1}^{3}=\alpha_{3} ; & q_{2}^{3}=\beta_{2} ; & q_{3}^{3}=\gamma+\pi_{2} ; & q_{4}^{3}=\delta+\beta-\beta_{2} \\
q_{1}^{4}=\alpha_{4} ; & q_{2}^{4}=\beta_{2}+\pi ; & q_{3}^{4}=\gamma+\pi_{2} ; & q_{4}^{4}=\delta+\beta-\beta_{2}+\pi
\end{array}
$$

When the optimal plan for the entire $n$-link manipulator is defined, it is required to fix all arbitrary variables. In case there is no joint limit, they can be set to any value. In contrast, in case the joint angles are limited, these variables should be properly selected using the following rules:

Rule 1. If the joint limits for the angle are narrower than $2 \pi / 3$ while plan of experiments requires higher width for joint variations, the joint coordinates of this joint should be changed with the one for which the joint coordinates can be set within the interval of the length $\pi$.

Rule 2. If the joint limits for the angle are narrower than $\pi$, some optimality conditions in (4) cannot be satisfied and the plan of experiments should be modified. In this case, the best results can be achieved when the joint coordinates are set to the joint limits.

Rule 3. For the joint with a range of variation equal to $\pi$, an arbitrary parameter can be fixed in the interval $\left[q_{\min } ; q_{\max }-\pi\right]$, where $q_{\min }$ and $q_{\max }$ are the corresponding joint limits.

Rule 4. For the joint with a range of variation equal to $2 \pi / 3$, an arbitrary parameter can be fixed in the interval $\left[q_{\min }+\pi / 3 ; q_{\max }-\pi / 3\right]$.

Hence, the above defined properties and rules allow us to generate optimal plan of experiments for the complex manipulator using simple patterns without any tedious computation. In the next section the obtained results will be applied for the generation of a plan of experiments for a 6-dof manipulator.

\section{Case study: optimal measurement poses for a 6-dof robot}

To show the efficiency of the developed optimal measurement pose selection technique, let us consider a 6-dof serial manipulator with six revolute joints and six links $\left(l_{1}=1.4 \mathrm{~m}, l_{2}=1.1 \mathrm{~m}, l_{3}=1.0 \mathrm{~m}, l_{4}=0.8 \mathrm{~m}, l_{5}=0.6 \mathrm{~m}, l_{6}=0.4 \mathrm{~m}\right)$, whose joint limits are $-140^{\circ} \leq q 1 \leq 0^{\circ},-120^{\circ} \leq q 2 \leq 90^{\circ},-120^{\circ} \leq q 3 \leq 120^{\circ}$, $-180^{\circ} \leq q 4 \leq 180^{\circ}, 0^{\circ} \leq q 5 \leq 210^{\circ}, 0^{\circ} \leq q 6 \leq 360^{\circ}$. In order to simplify the determination of measurement configurations, the manipulator can be split into two sub-chains: (i) 3-link chain corresponding to links \#1-\#3, and (ii) 4-link chain composed of a virtual link/joint and links \#4 - \#6 of entire manipulator. 
According to the geometrical patterns presented above, the optimal plans for the first and second sub-chains can be generated using Eqs. (7) and (9), respectively. For the entire manipulator, the optimal plan can be obtained using Property 3 (it consists of 12 measurement configurations). However, such a plan of experiments requires a large range of variations for $q_{2}$ (equal to $2 \pi / 3$ ), which cannot be included in the joint limits. This difficulty can be overcome using Property 2 allowing us permutation of $q_{2}$ and $q_{4}$. This provides us with the following plan of calibration experiments, which satisfies Eq. (4) and, consequently, insures the covariance matrix diagonality:

$$
\begin{array}{cccccc}
\alpha_{1} ; & \delta_{1} ; & \gamma & \beta ; & \chi ; & \varphi \\
\alpha_{1} ; & \delta_{1}+\pi ; & \gamma & \beta ; & \chi ; & \varphi+\pi \\
\alpha_{1} ; & \delta_{2} ; & \gamma & \beta ; & \chi+\pi_{2} ; & \varphi+\delta_{1}-\delta_{2} \\
\alpha_{1} ; & \delta_{2}+\pi ; & \gamma & \beta ; & \chi+\pi_{2} ; & \varphi+\delta_{1}-\delta_{2}+\pi \\
\alpha_{2} ; & \delta_{1} ; & \gamma+2 \pi / 3 & \beta+2 \pi / 3 ; & \chi ; & \varphi \\
\alpha_{2} ; & \delta_{1}+\pi ; & \gamma+2 \pi / 3 & \beta+2 \pi / 3 ; & \chi ; & \varphi+\pi \\
\alpha_{2} ; & \delta_{2} ; & \gamma+2 \pi / 3 & \beta+2 \pi / 3 ; & \chi+\pi_{2} ; & \varphi+\delta_{1}-\delta_{2} \\
\alpha_{2} ; & \delta_{2}+\pi ; & \gamma+2 \pi / 3 & \beta+2 \pi / 3 ; & \chi+\pi_{2} ; & \varphi+\delta_{1}-\delta_{2}+\pi \\
\alpha_{3} ; & \delta_{1} ; & \gamma-2 \pi / 3 & \beta-2 \pi / 3 ; & \chi ; & \varphi \\
\alpha_{3} ; & \delta_{1}+\pi ; & \gamma-2 \pi / 3 & \beta-2 \pi / 3 ; & \chi ; & \varphi+\pi \\
\alpha_{3} ; & \delta_{2} ; & \gamma-2 \pi / 3 & \beta-2 \pi / 3 ; & \chi+\pi_{2} ; & \varphi+\delta_{1}-\delta_{2} \\
\alpha_{3} ; & \delta_{2}+\pi ; & \gamma-2 \pi / 3 & \beta-2 \pi / 3 ; & \chi+\pi_{2} ; & \varphi+\delta_{1}-\delta_{2}+\pi
\end{array}
$$

Taking into account given joint limits, the arbitrary angles in the above expression have been set as

$$
\begin{array}{cccc}
\alpha_{1}=0 ; & \alpha_{2}=-\pi / 3 ; & \alpha_{3}=-2 \pi / 3 ; & \beta=0 ; \\
\delta_{1}=-2 \pi / 3 ; & \delta_{2}=-\pi / 2 ; & \gamma=0 ; & \chi=\pi / 6 ; \quad \varphi=\pi / 2
\end{array}
$$

Using this plan of experiments, the desired geometrical parameters can be identified with a precision of $0.029 \mathrm{~mm}$ for the link lengths and 4.13 mdeg for the joint offsets (assuming that the measurement system precision is equal to $0.1 \mathrm{~mm}$ ). In more details, relevant results are presented in Table 1. For comparison purposes, the identification accuracy has been also evaluated for regular and random plans of experiments that contain the same number of measurement configurations. It should be noted that for a 6-dof manipulator it is not possible to obtain a regular plan with 12 measurement configurations. For this reason the plan has been generated using 5 measurement configurations within the joint limits from which 12 configurations have been selected randomly. In order to reduce the factor of particular set of measurement configurations, simulations for regular and random plans have been repeated 1000 times and the results have been averaged.

Hence, simulation results confirm advantages of the proposed approach. In this study, the identification accuracy for the optimal plan is better by $34-72 \%$ comparing with the random plan and by $38-48 \%$ compared with the regular plan of experiments. In addition, maximum positioning errors have been reduced by $44 \%$ and $57 \%$, respectively. It should be stressed that the proposed approach does not require 
Table 1 Identification accuracy for different plans of calibration experiments.

\begin{tabular}{ccccccc}
\hline Joint & \multicolumn{2}{c}{ Proposed plan } & \multicolumn{2}{c}{ Regular plan } & \multicolumn{2}{c}{ Random plan } \\
& $\delta q_{i},[\mathrm{mdeg}]$ & $\delta L_{i},[\mathrm{~mm}]$ & $\delta q_{i},[\mathrm{mdeg}]$ & $\delta L_{i},[\mathrm{~mm}]$ & $\delta q_{i},[\mathrm{mdeg}]$ & $\delta L_{i},[\mathrm{~mm}]$ \\
\hline 1 & 1.18 & 0.029 & 1.70 & 0.041 & 1.90 & 0.046 \\
2 & 1.50 & 0.029 & 2.21 & 0.042 & 2.58 & 0.050 \\
3 & 1.65 & 0.029 & 2.32 & 0.041 & 2.46 & 0.043 \\
4 & 2.07 & 0.029 & 3.04 & 0.042 & 3.32 & 0.046 \\
5 & 2.76 & 0.029 & 4.04 & 0.042 & 4.39 & 0.046 \\
6 & 4.13 & 0.029 & 5.73 & 0.040 & 5.55 & 0.039 \\
\hline$\rho_{\max }$ & \multicolumn{2}{c}{0.61} & \multicolumn{2}{c}{0.88} & \multicolumn{3}{c}{0.96} \\
\hline
\end{tabular}

any computation for optimal measurement pose selection and is able to improve the identification accuracy using a small number of measurement configurations.

\section{Conclusions}

The paper presented a new approach for the design of calibration experiments for robotic manipulators that essentially simplifies the optimal pose selection procedure. The main theoretical results are expressed as a set of several properties and rules, which allow user to obtain optimal measurement configurations without any computation, simply using superpositions and permutations of the proposed geometrical patterns describing optimal measurement configurations for 2-, 3- and 4link manipulators. The efficiency of the developed approach has been confirmed by an illustrative example that deals with the calibration of a 6-dof manipulator.

Acknowledgements The work presented in this paper was partially funded by the project ANR (Project ANR-2010-SEGI-003-02-COROUSSO), France and FEDER ROBOTEX, France.

\section{References}

1. Atkinson, A.C., Donev, A.N., Tobias, R.D.: Optimum experimental designs, with SAS. Oxford University Press Oxford (2007)

2. Borm, J.H., Meng, C.H.: Determination of optimal measurement configurations for robot calibration based on observability measure. The International Journal of Robotics Research 10(1), 51-63 (1991)

3. Elatta, A., Gen, L.P., Zhi, F.L., Daoyuan, Y., Fei, L.: An overview of robot calibration. Information Technology Journal 3(1), 74-78 (2004)

4. Hollerbach, J., Khalil, W., Gautier, M.: Model identification. In: B. Siciliano, O. Khatib (eds.) Springer Handbook of Robotics, pp. 321-344. Springer Berlin Heidelberg (2008)

5. Klimchik, A., Wu, Y., Caro, S., Pashkevich, A.: Design of experiments for calibration of planar anthropomorphic manipulators. In: Advanced Intelligent Mechatronics (AIM), 2011 IEEE/ASME International Conference on, pp. 576-581. IEEE (2011)

6. Mooring, B.W., Roth, Z.S., Driels, M.R.: Fundamentals of manipulator calibration. Wiley New York (1991) 